\section{Septic thrombophlebitis with persistent methicillin-resistant Staphylococcus aureus bac- teremia and de novo resistance to vancomycin and daptomycin}

\author{
Yael Koton, ${ }^{1,2}$ Zafrir Or, ${ }^{3}$ \\ Naiel Bisharat ${ }^{1,2}$ \\ ${ }^{1}$ Department of Medicine D, Emek \\ Medical Center, Afula; ${ }^{2}$ Ruth and Bruce \\ Rappaport Faculty of Medicine, \\ Technion - Israel Institute of \\ Technology, Haifa; ${ }^{3}$ Department of \\ Cardiology, Emek Medical Center, \\ Afula, Israel
}

\begin{abstract}
Persistent methicillin-resistant Staphylococcus aureus (MRSA) bacteremia is associated with significant risk of mortality, especially when it occurs while on appropriate antimicrobial therapy. We herein describe an unusual case of a patient with prosthetic aortic tissue valve, who suffered from central venous catheter related MRSA bacteremia with septic thrombus formation in the superior vena cava. MRSA bacteremia persisted despite removal of the catheter and appropriate antimicrobial therapy including vancomycin, rifampin, and daptomycin. Subsequently, the MRSA strain exhibited de novo resistance to vancomycin, rifampin and daptomycin. Eventually, salvage combination therapy with high dose daptomycin and trimethoprim-sulfamethoxazole was successful and achieved clearance of MRSA bacteremia The case illustrates the growing complexity of treating MRSA infections.
\end{abstract}

\section{Introduction}

Persistent methicillin-resistant Staphylococcus aureus (MRSA) bacteremia is a challenging clinical problem that carries a significant risk for mortality and raises concerns because it develops despite the administration of appropriate antimicrobial therapy. ${ }^{1}$ Previous studies have identified several risk factors that are associated with the development of persistent MRSA bacteremia such as endocarditis, retained central venous catheters, metastatic infection, and congestive heart failure. ${ }^{1,2}$ We describe a patient who suffered from persistent MRSA bacteremia secondary to septic thrombus formation in the superior vena cava (SVC) with emergence of resistance to vancomycin and daptomycin while on therapy.

\section{Case Report}

A 50-year-old man was admitted to the emergency department due to high spiking fever and disorientation. His past medical history was remarkable for antiphospholipid syndrome with previous episodes of deep vein thrombosis and ischemic stroke, for which he was treated with warfarin. In addition, he underwent prosthetic tissue aortic valve replacement due to aortic stenosis. Three months prior to present admission he was admitted to another hospital due to brainstem hemorrhage that was complicated with nosocomial pneumonia and severe renal failure for which he received hemodialysis via permacath. Anticoagulant therapy was switched from warfarin to subcutaneous enoxaparin upon partial recovery of his kidney function. The permacath was retained until achievement of full recovery of kidney function. The patient did not attend a scheduled appointment for permacath removal. Vital signs upon admission showed a temperature of $39^{\circ} \mathrm{C}$, blood pressure of $80 / 50 \mathrm{mmHg}$, pulse of 128 beats per minute, 36 breaths per minute, and oxygen saturation of $88 \%$ on room air. His physical examination was remarkable for disorientation without any obvious foci of infection, including entry site of the permacath. Blood tests showed leukocytosis of 20,000 per cubic millimeter, hemoglobin concentration of $12 \mathrm{~g} / \mathrm{dL}$, platelets count of 550,000 per cubic millimeter, blood urea nitrogen and creatinine levels were slightly elevated. His chest xray was normal, urine analysis showed microscopic hematuria, blood and urine were drawn for culture. The permacath was extracted and the patient was treated with vancomycin, piperacillin-tazobactam, amikacin, vasopressors and admitted to intensive care unit (ICU). His blood cultures grew MRSA susceptible to vancomycin with a minimal inhibitory concentration (MIC) of $0.75 \mu \mathrm{g} / \mathrm{mL}$, daptomycin ( $\mathrm{MIC}=0.5 \mu \mathrm{g} / \mathrm{mL}$ ), rifampin, and trimethoprim-sulfamethoxazole (TMP/SMX), cultures from the tip of permacath also yielded MRSA (Table 1). Antimicrobial susceptibility testing was carried out using the KirbyBauer methods or E-test according to the Clinical and Laboratory Standards Institute (CLSI) guidelines. Transthoracic echocardiogram showed a normal function of the prosthetic valve. The patient's condition stabilized and after $48 \mathrm{~h}$ stay in ICU he was transferred to a medical ward. The patient
Corresponding: Naiel Bisharat, Department of Medicine D, Emek Medical Center, 18 Rabin Avenue, Afula 18341, Israel.

Tel.: +1.972.4.6494520 - Fax: +1.972.4.6494518. E-mail: bisharat_na@clalit.org.il

Key words: Thrombosis; superior vena cava; methicillin-resistant Staphylococcus aureus; drug resistance.

Contributions: YK wrote the first draft, $\mathrm{ZO}$ assisted in writing the manuscript and obtained the images, NB supervised the work and wrote the final draft.

Conflict of interest: the authors declare no potential conflict of interest.

Received for publication: 17 December 2016. Revision received: 29 January 2017.

Accepted for publication: 30 January 2017.

This work is licensed under a Creative Commons Attribution-NonCommercial 4.0 International License (CC BY-NC 4.0).

(C) Copyright Y. Kotton et al., 2017

Licensee PAGEPress, Italy

Infectious Disease Reports 2017; 9:7008

doi:10.4081/idr.2017.7008

was treated with vancomycin and rifampin but his fever persisted and blood cultures grew MRSA again. The MRSA strain was susceptible to vancomycin $(\mathrm{MIC}=1 \mu \mathrm{g} / \mathrm{ml})$ but resistant to rifampin (Table 1). Antimicrobial therapy was switched to vancomycin plus piperacillin-tazobactam (Table 2) but without success. Transesophageal echocardiogram (TEE) showed a thrombus measuring $8 \mathrm{~mm}$ (width) by 8 $\mathrm{mm}$ (length) in the SVC, approximately 2.2 $\mathrm{cm}$ before entry into the right atrium (Figure 1). A whole body computed tomography scan failed to show any other foci of infection. The antimicrobial therapy was switched to daptomycin $(10 \mathrm{mg} / \mathrm{kg})$ plus oxacillin, but the patient was still bacteremic with MRSA. However, the strain became non-susceptible to daptomycin ( $\mathrm{MIC}=1.5 \mu \mathrm{g} / \mathrm{mL}$ ) and resistant to vancomycin $(\mathrm{MIC}>2 \mu \mathrm{g} / \mathrm{mL})$. At this stage, daptomycin dose was increased to maximum $(12 \mathrm{mg} / \mathrm{kg})$ and intravenous TMP/SMX was added to daptomycin (Table 2), this combination treatment eventually yielded a satisfactory response with clearance of MRSA bacteremia within 3 days. The patient continued treatment with enoxaparin and maintained therapeutic anti Xa levels, his condition stabilized and serial blood cultures were sterile. Unfortunately, one day prior to a scheduled TEE study the patient suffered a cardiac arrest with an ini- 
tial cardiac rhythm of asystole and eventually died. Molecular characterization ( $\mathrm{mul}$ tilocus sequence typing and spa typing), carried out as previously described, ${ }^{3}$ showed that all MRSA strains obtained throughout the patient's hospitalization belonged to the same genotype (sequence type 5, spa type t002).

\section{Discussion}

Septic thrombus formation is a condition characterized by venous thrombosis, inflammation, and bacteremia. ${ }^{4}$ Septic thrombophlebitis can affect peripheral, central, portal, pelvic, or intracranial venous sinuses. Septic thrombophlebitis of the SVC is mainly associated with central venous catheter placement; ${ }^{5}$ other causes include burns, hyper-coagulable conditions, and administration of total parenteral nutrition. $^{6-8}$ Successful treatment includes removal of any indwelling catheters combined with appropriate antimicrobial therapy. The patient described above suffered from MRSA bacteremia that persisted despite removal of central venous catheter and appropriate antimicrobial therapy.

Most cases of SVC thrombosis are related to the presence of indwelling intravascular devices, malignancy, and hypercoagulable conditions, ${ }^{9}$ two of which existed in the current case. The preferred management of asymptomatic and hemodynamically stable patients is anticoagulation, while thrombectomy and thrombolysis are indicated in massive thrombosis with hemo- dynamic instability, and pulmonary embolism. ${ }^{10}$ Balloon angioplasty and stent insertion is usually indicated in patients with chronic obstructions and SVC syndrome. Treatment of persistent MRSA bacteremia is a challenging clinical problem especially with emergence of resistance while on therapy and subsequent clinical failure. In the current case, the MRSA strain became resistant to vancomycin, rifampin, and daptomycin during therapy but the patient was successfully treated using salvage combination therapy with high dose daptomycin and TMP/SMX. Development of de novo resistance to vancomycin and daptomycin during therapy of MRSA infections occurs infrequently. ${ }^{11-13}$ The exact mechanisms by which MRSA strains develop resistance to daptomycin during vancomycin therapy are unclear, it has been suggested that reduced susceptibility to daptomycin is associated with transition of vancomycin-susceptible phenotype to a vancomycin-intermediate $S$. aureus (VISA) phenotype through a vancomycin-heteroresistant S. aureus (hVISA) intermediary. ${ }^{14-16}$ Nevertheless, emergence of daptomycin nonsusceptibility has also been documented in the absence of vancomycin exposure. ${ }^{12}$ The mechanism of daptomycin nonsuscep-

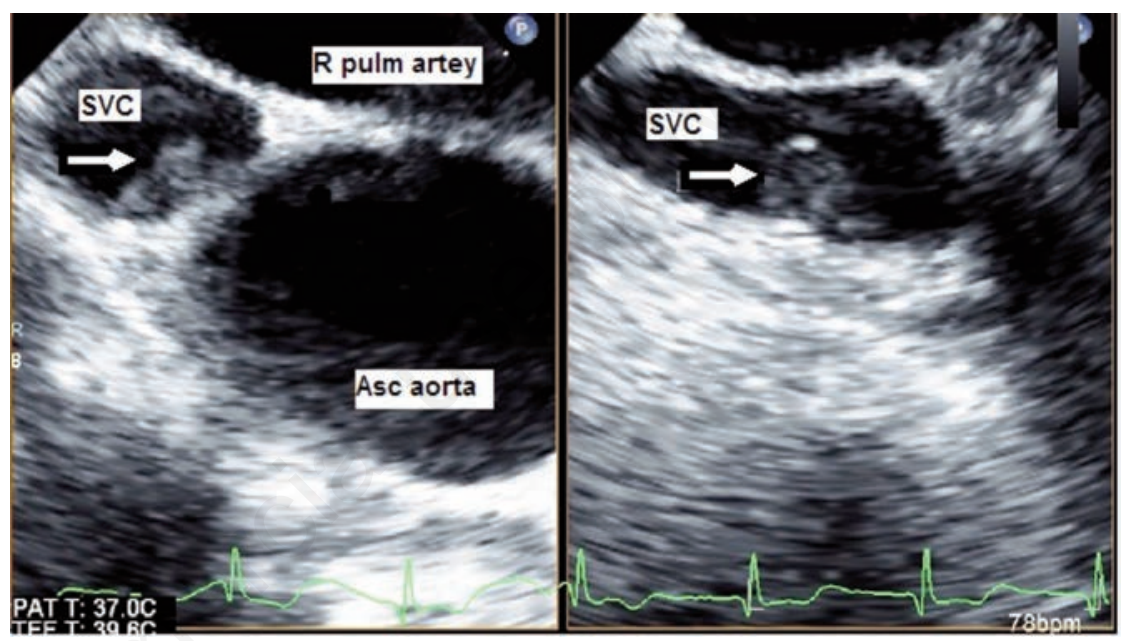

Figure 1. Great vessels view of trans-esophageal echocardiogram showing thrombus (arrow) in superior vena cava (SVC), ascending aorta, and right pulmonary artery.

Table 1. Susceptibility testing of MRSA isolates during therapy with various antimicrobial agents.

\begin{tabular}{|c|c|c|c|c|c|c|}
\hline Sample date & Vancomycin, MIC, mg/L & Rifampin & Gentamycin & Linezolid & TMP/SMX & Daptomycin, MIC, mg/L \\
\hline Day 1 & $1(\mathrm{~S}) \mathrm{c}$ & S & $\mathrm{R}$ & $0.75(\mathrm{~S})$ & S & $0.75(\mathrm{~S})$ \\
\hline Day 5 & $1.5(\mathrm{~S})$ & $\mathrm{R}$ & $\mathrm{R}$ & $0.75(\mathrm{~S})$ & S & $0.75(\mathrm{~S})$ \\
\hline Day 7 & $1.5(\mathrm{~S})$ & $\mathrm{R}$ & $\mathrm{R}$ & $0.75(\mathrm{~S})$ & S & $1(\mathrm{~S})$ \\
\hline Day 10 & $>2(\mathrm{R})$ & $\mathrm{R}$ & $\mathrm{R}$ & $0.75(\mathrm{~S})$ & S & $1.5(\mathrm{R})$ \\
\hline
\end{tabular}

MIC, minimal inhibitory concentration; TMP/SMX, trimethoprim-sulfamethoxazole; S, susceptible; R. resistant.

Table 2. Antimicrobial agents administered during hospital stay along with MRSA bacteremia status.

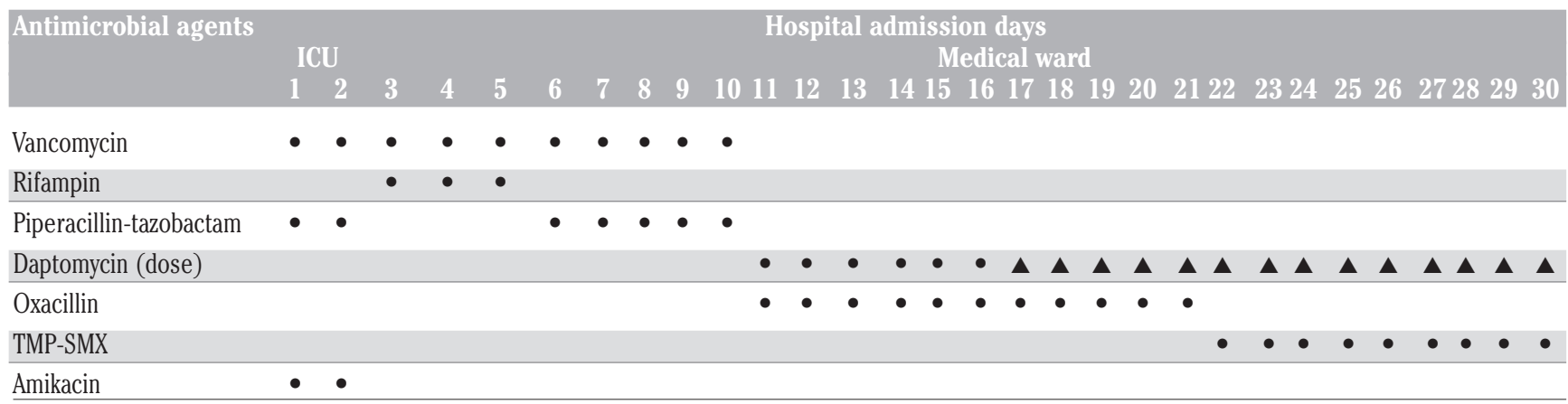

MRSA bacteremia status: from Day 1 to Day 24. For daptomycin: • dose $10 \mathrm{mg} / \mathrm{kg}$; $\mathbf{\Delta}$ dose $12 \mathrm{mg} / \mathrm{kg}$. 
tibility in $S$. aureus is not completely understood, but has been linked to alterations in cell wall thickening, alterations in surface charge, membrane phospholipid asymmetry, and drug binding. ${ }^{17}$

There is a growing concern regarding clinical failure of treatment for complicated MRSA infections. Treatment options for persistent MRSA bacteremia or bacteremia due to VISA or vancomycin-resistant S.aureus (VRSA), include vancomycinbased therapy with semisynthetic penicillins, cephalosporins, ${ }^{5} \quad \beta$-lactam $/ \beta$-lactamase inhibitor combinations, or carbapenems. ${ }^{18}$ It has been suggested that synergy results from a reduction in cell wall thickness caused by $\beta$-lactam exposure with a reduction in sequestration of the glycopeptides. Similarly, daptomycin-based therapy was evaluated using combination with $\beta$ lactams, ceftaroline, and TMP/SMX. ${ }^{19,20}$ To date, several other combinations have been evaluated including ceftaroline-based therapy, linezolid-based therapy, quinupristin/ dalfopristin, telavancin, and trimethoprim/ sulfamethoxazole-based therapy. ${ }^{19}$ Current recommendations suggest using combination antibiotic therapy for salvage treatment of MRSA bacteremia. ${ }^{19}$ Despite the fact that the MRSA strain was persistently susceptible to linezolid we chose not to administer a linezolid-based salvage therapy for two reasons. First, unlike vancomycin and daptomycin, linezolid is bacteriostatic, and second, a previous study showed that in patients with persistent MRSA bacteremia while receiving vancomycin, a switch to linezolid therapy did not lead to better outcomes than those in whom vancomycin was continued. ${ }^{21}$

\section{Conclusions}

The case described exemplifies the growing complexity of treating MRSA infections especially when do novo resistance to antimicrobial therapy evolves during therapy. Lastly, the patient succumbed to death few days after clearance of MRSA bacteremia, autopsy was refused by the patient's family. We hypothesize that his death was caused by dislodgement of the SVC thrombus to the pulmonary trunk causing massive pulmonary embolus. Notwithstanding the tragic outcome and given his hemodynamically stable condition and lack of signs of SVC syndrome, we believe that the decision to treat him only with anticoagulation while maintaining therapeutic anti Xa levels was sensible and in accordance with current guidelines. ${ }^{10}$

\section{References}

1. Neuner EA, Casabar E, Reichley R, McKinnon PS. Clinical, microbiologic, and genetic determinants of persistent methicillin-resistant Staphylococcus aureus bacteremia. Diagn Microbiol Infect Dis 2010;67:228-33.

2. Ok HS, Lee HS, Park MJ, et al. Predictors and clinical outcomes of persistent methicillin-resistant Staphylococcus aureus bacteremia: a prospective observational study. Korean J Intern Med 2013;28:678-86.

3. Eghbaria S, Schnitser K, Danial-Farran $\mathrm{N}$, et al. Molecular characterization of methicillin-resistant Staphylococcus aureus strains isolated from patients with hospital readmissions. J Clin Microbiol 2014;52:1256-8.

4. Mermel LA, Allon M, Bouza E, et al. Clinical practice guidelines for the diagnosis and management of intravascular catheter-related infection: 2009 Update by the Infectious Diseases Society of America. Clin Infect Dis 2009;49:1-45.

5. Barber KE, Rybak MJ, Sakoulas G. Vancomycin plus ceftaroline shows potent in vitro synergy and was successfully utilized to clear persistent daptomycin-non-susceptible MRSA bacteraemia. J Antimicrob Chemother 2014; 70:311-3.

6. Viale P, Stefani S. Vascular catheterassociated infections: a microbiological and therapeutic update. J Chemother 2006; 18:235-49.

7. Ferreira A, Bettencourt Fernando PM, Capucho R, Macedo F. Total parenteral nutrition by central venous catheter complicated by right atrial septic thrombus. Postgrad Med J 1994;70:520.

8. Tzortzis S, Apostolakis S, Xenakis K, et al. Catheter-related septic thrombophlebitis of the superior vena cava involving the atrial septum: a case report. Cases J 2008;1:272.

9. Rice TW, Rodriguez RM, Light RW. The superior vena cava syndrome: clinical characteristics and evolving etiology. Medicine (Baltimore) 2006;85:37-42.

10. Burns KE, McLaren A. Catheter-related right atrial thrombus and pulmonary embolism: a case report and systematic review of the literature. Can Respir J 2009; 16:163-5.

11. Twele L, Moyen E, Zhang K, et al. Methicillin-resistant Staphylococcus aureus endocarditis and de novo development of daptomycin resistance during therapy. Can J Infect Dis Med Microbiol 2011;21:89-93.

12. Fowler VG, Jr., Boucher HW, Corey GR, et al. Daptomycin versus standard therapy for bacteremia and endocarditis caused by Staphylococcus aureus. N Engl J Med 2006;355:653-65.

13. Lee $\mathrm{CH}$, Wang MC, Huang IW, et al. Development of daptomycin nonsusceptibility with heterogeneous vancomycin-intermediate resistance and oxacillin susceptibility in methicillinresistant Staphylococcus aureus during high-dose daptomycin treatment. Antimicrob Agents Chemother 2010;54:4038-40.

14. van Hal SJ, Paterson DL, Gosbell IB. Emergence of daptomycin resistance following vancomycin-unresponsive Staphylococcus aureus bacteraemia in a daptomycin-naive patient - a review of the literature. Eur J Clin Microbiol Infect Dis 2010;30:603-10.

15. Cui L, Tominaga E, Neoh HM, Hiramatsu K. Correlation between reduced daptomycin susceptibility and vancomycin resistance in vancomycinintermediate Staphylococcus aureus. Antimicrob Agents Chemother 2006;50:1079-82.

16. Patel JB, Jevitt LA, Hageman J, et al. An association between reduced susceptibility to daptomycin and reduced susceptibility to vancomycin in Staphylococcus aureus. Clin Infect Dis 2006;42:1652-3.

17. Jones T, Yeaman MR, Sakoulas G, et al. Failures in clinical treatment of Staphylococcus aureus Infection with daptomycin are associated with alterations in surface charge, membrane phospholipid asymmetry, and drug binding. Antimicrob Agents Chemother 2008;52:269-78.

18. Holubar M, Meng L, Deresinski S. Bacteremia due to methicillin-resistant Staphylococcus aureus: new therapeutic approaches. Infect Dis Clin North Am 2016;30:491-507.

19. Kullar R, Sakoulas G, Deresinski S, van Hal SJ. When sepsis persists: a review of MRSA bacteraemia salvage therapy. J Antimicrob Chemother 2016;71:57686.

20. Claeys KC, Smith JR, Casapao AM, et al. Impact of the combination of daptomycin and trimethoprim-sulfamethoxazole on clinical outcomes in methicillinresistant Staphylococcus aureus infections. Antimicrob Agents Chemother 2015;59:1969-76.

21. Park HJ, Kim SH, Kim MJ, et al. Efficacy of linezolid-based salvage therapy compared with glycopeptidebased therapy in patients with persistent methicillin-resistant Staphylococcus aureus bacteremia. J Infect 2012;65: 505-12. 